\title{
EXTENDING THE LINEAR QUADRATIC GAUSSIAN / LOOP TRANSFER RECOVERY TECHNIQUE TO A CLASS OF DISTRIBUTED PARAMETER SYSTEMS
}

\author{
Randall N. Paschall and Alan V. Lair \\ Air Force Institute of Technology \\ Department of Electrical and Computer Engineering \\ Wright-Patterson AFB, Ohio 45433
}

28th CDC

\begin{abstract}
Sufficient conditions for extending the Linear Quadratic Gaussian / Loop Transfer Recovery technique to a class of distributed parame ter systems are presented. Two different approaches are considered in developing the condition. The goa is to fully extend the technique to a class of infinite dimensional regulator problems which utilize Kalman filter. It is not known whether or not the sufficient conditions are satisfied by the entire class of problems considered. The paper will discuss difficulties encountered in trying to prove that the sufficient conditions are satisfied, and will present an approximation based on the work of H.T. Banks.
\end{abstract}

\section{Introduction}

This paper will consider the LQG/LTR technique for robustness enhancement. As developed by Doyle and Stein [1] the technique involves adding a pseudonoise term $\beta^{2} B V B^{*}$ to the operator $Q_{f}$ in the filter algebraic Ricatti equation (A.R.E.), and tuning the equation so as to recover, asymptotically, the loop transfer function of the LQ regulator. The technique was extended to a class of distributed parameter systems by Matson [2], but not to the entire class of systems considered in this paper. Specifically, Matson extended the technique to those problems for which $A^{*}$ is bounded (which includes finite-dimensional problems), and those problems where $R(B) \cup R\left(Q_{\jmath}\right)$ is contained in a finite-dimensional space spanned by a finite number of eigenfunctions of $A^{*}$ (see [2] Lemma 4.11).

The class of problems addressed in this paper is the set of infinitedimensional systems of the form

$$
\begin{gathered}
\dot{x}(t)=A x(t)+B u(t)+G w(t), \quad x_{0}=x(0) \in D(A) \\
y(t)=C x(t)+\eta(t)
\end{gathered}
$$

where the control vector $u$ is in the input space $U=L^{2}\left\{[0, \infty) ; \Re^{N}\right\}, x$ is an element of a Hilbert space $\mathcal{H}, y$ is an observation vector which is an element the output space $Y=\Re^{N}$, and $w$ and $\eta$ are white Gaussian noise terms with realizations in the spaces $Y$ and $\mathcal{H}$ respectively. The strength of the dynamics noise term $w$ is described by the positive semi-definite operator $Q_{o}$, and the strength of the measurement noise term $\eta$ is described by the strictly positive operator $R_{f}$ (these operators will be discussed more in Chapter 2). The operator $Q_{f}$ used in the Kalman filter design will be chosen so that $Q_{f}=G Q_{o} G^{*}$, where $G^{*}$ denotes the adjoint of $G . x(t)$ will be denoted simply as $x$ (and similarly for the other functions), and the following assumptions are made:

1. $A$ is the infinitesimal generator of a $C_{0}$ semigroup (i.e. strongly continuous) $T(t)$ on a real separable Hilbert space (Hilbert space with a countable orthonormal basis) $\mathcal{H}$ [3].

2. $B$ is a bounded linear operator from $\Re^{N}$ to $\mathcal{H}$.

3. $C$ is a bounded linear operator from $\mathcal{H}$ to $\Re^{N}$.

4. $G$ is a bounded linear operator from the Hilbert space $\mathcal{H}$ to $\mathcal{H}$

5. The spectrum of $A$ (denoted $\sigma(A)$ ) is discrete.

6. The system is exponentially stabilizable and detectable [3].

7. The eigenvectors of $A$ are complete.

8. The system is minimum phase (i.e. no transmission zeros in the righthalf plane).

9. A satisfies the spectrum decomposition assumption [4].

10. The restriction of $A$ to the stable subspace $\mathcal{H}_{\text {, satisfies the spectrum }}$ determined growth assumption [3] and generates an exponentially sta- ble semigroup.

11. The number of inputs equals the number of outputs.

The steady state Kalman filter constant gain operator $K_{f}$ is given by

$$
K_{f}=P_{f} C^{*} R_{f}^{-1}
$$

where the covariance operator $P_{f}$ is the unique positive self-adjoint solution to the following A.R.E.

$$
\left\langle P_{f} h, A^{*} k\right\rangle+\left\langle A^{*} h, P_{j} k\right\rangle+\left\langle Q_{f} h, k\right\rangle=\left\langle P_{j} C^{*} R_{j}^{-1} C P_{j} h, k\right\rangle
$$

for all $h, k \in D\left(A^{*}\right)$, under the standard assumption that $(A, G)$ is stabilizable and $(A, C)$ is detectable. Also, note that $Q_{\rho}=G Q_{0} G^{*}$, where $Q_{0}$ is the positive semi-definite operator that describes the strength of the dynamics driving noise $w$.

The LQG/LTR technique modifies the noise term so that $Q_{J}=Q_{J_{0}}+$ $\beta^{2} B V B^{*}$ where $Q_{\text {/。 }}$ is the nominal value of $Q$, that provides the best filter tuning at design conditions, $\beta$ is a positive scalar, $B$ is the system input operator, and $V$ is any $\mathbf{N}$ by $\mathbf{N}$ positive definite matrix. This then yields a family of Kalman filter gain operators $K_{J \beta}$ which are given by

$$
K_{f \beta}=P_{\beta} C^{*} R_{f}^{-1}
$$

and $P_{\beta}$ is the solution of the algebraic Riccati equation

$\left(P_{\beta} h, A^{*} k\right\rangle+\left\langle A^{*} h, P_{\beta} k\right\rangle+\left\langle\left(Q_{j}+\beta^{2} B V B^{*} h, k\right\rangle=\left\langle P_{\beta} C^{*} R_{j}^{-i} C P_{\beta} h, k\right)\right.$

This A.R.E. can be written in terms of the operator $Y_{\beta}$ by setting $Y_{\beta}=\frac{(6)}{P_{\theta}}$. This yields

$\left\langle Y_{\beta} h, A^{*} k\right\rangle+\left\langle A^{*} h, Y_{\beta} k\right\rangle+\left\langle\left(B V B^{*}+\beta^{-2} Q_{f}\right) h, k\right\rangle=\beta^{2}\left\langle Y_{\beta} C^{*} R_{f}^{-1} C Y_{\beta} h, k\right\rangle$

for all $h, k \in D\left(A^{*}\right)$.

The objective of the LQG/LTR technique is to increase the scalar $\beta$ (and thus the noise term $Q_{f}$ ) so that the following limit exists:

$$
\lim _{\beta \rightarrow \infty} K_{f \beta}\left[I+C(s I-A)^{-1} K_{f \beta}\right]^{-1} r=B\left[C(s I-A)^{-1} B\right]^{-1} r \quad \forall r \in \mathcal{C}^{N}
$$

If this limit exists, then one is able to recover the loop transfer function associated with the $L Q$ regulator at the input to the plant (or by using the dual procedure of Kwakernaak and Sivan [5], one can recover the loop transfer function associated with the Kalman filter at the output of the plant). Matson proved that a sufficient condition for this limit to exist is if

$$
\beta^{2} Y_{\beta} C^{*} R_{J}^{-1} C Y_{\beta} \stackrel{s}{\rightarrow} B V B^{*} \quad \text { as } \beta \rightarrow \infty
$$

where $\stackrel{s}{\rightarrow}$ denotes strong convergence. A sequence of bounded linear operators $K_{\theta}$ is said to converge strongly to a bounded linear operator $K$ if $\lim _{\beta \rightarrow \infty}\left\|\left(K_{\beta}-K^{\prime}\right) h\right\|=0$ for each $h$ in the normed linear space on which $K$ is defined. Other types of convergence include weak and uniform (see [6] for a discussion of these).

This paper looks at conditions under which this strong convergence exists. Section 2 provides one set of sufficient conditions. Section 3 explains why many authors have not worried about strong convergence, and then a second set of sufficient conditions is provided. Since neither of the suffcient conditions developed in this paper are physically motivated, Section 4 considers how to approximate the LQG/LTR technique using the approach developed by Banks [i].

\section{Sufficient Condition}

The following theorem gives a sufficient condition for the results of Matson [2] to be valid for the class of systems considered in this research. 
THEOREM 2.1: Let $\mathcal{H}$ be a real Hilbert space and $Y_{\beta} \in \mathcal{L}(\mathcal{K})$ be the unique, positive, self-adjoint solution to the algebraic Riccati equation

$\left\langle Y_{\beta} h, A^{*} k\right\rangle+\left\langle A^{*} h, Y_{\beta} k\right\rangle+\left\langle\left(B V B^{*}+\beta^{-2} Q_{J}\right) h, k\right\rangle=\beta^{2}\left\langle Y_{\beta} C^{*} R_{J}^{-1} C Y_{\beta} h, k\right\rangle$

for all $h, k \in D\left(A^{*}\right)$ where $A, B, Q_{f}, C$, and $R_{f}$ are as described in Section 1. Let $V$ be a positive definite $N$ by $N$ matrix, and let $\beta$ be a scalar $\geq 0$. Also, assume that the number of inputs equals the number of outputs. Then,

$$
\lim _{\beta \rightarrow \infty} \beta^{2}\left\langle Y_{\beta} C^{*} R_{f}^{-1} C Y_{\beta} h, k\right\rangle=\left\langle B V B^{*} h, k\right\rangle \quad \forall h, k \in D\left(A^{*}\right)
$$

implies that

$$
\beta^{2} Y_{\beta} C^{*} R_{f}^{-1} C Y_{\beta} \stackrel{s}{\rightarrow} B V B^{*} \quad \text { as } \beta \rightarrow \infty
$$

if the operator

$$
K_{\beta}=\left(B V B^{*}+\beta^{-2} Q_{f}-\beta^{2} Y_{\beta} C^{*} R_{f}^{-1} C Y_{\beta}\right)
$$

is uniformly bounded (in the operator norm) independent of $\beta$, for $\beta$ sufficiently large, and if $K_{\beta}$ is positive semi-definite for $\beta$ large.

The difficulty with this theorem is that, the conditions on $K_{\beta}$ are not physically motivated. There is not a known class of systems which satisfy these conditions by the nature of their state space operators. To use this theorem requires one to confirm the conditions on a problem by problem basis. However, it is this author's opinion that the conditions will be satisfied for a large class of problems. The boundedness assumption on $K_{\beta}$ is reasonable because the operator $Y_{\beta}$ is bounded independent of $\beta$. Thus, $h_{\beta}$ appears to be uniformly bounded as well. The proof of the theorem is now presented.

Proof: Since the system is assumed to be stabilizable and detectable, then as shown in Jacobson [8] and Curtain [9], this implies that the system operator $A$ satisfie the spectrum decomposition assumption and has finitely many unstable eigenvalues of finite multiplicity. Let $\mathcal{H}_{u}$ be the subspace spanned by the eigenfunctions corresponding to the unstable eigenvalues, and $\mathcal{H}$, be the subspace spanned by the eigenfunctions corresponding to the stable eigenvalues. Then as shown in Schumacher [4], one can write $\mathcal{H}=\mathcal{H}_{u} \oplus \mathcal{H}$, and as such one can decompose the system operators as follows:

$$
\begin{gathered}
A=\left[\begin{array}{cc}
A_{s} & 0 \\
0 & A_{u}
\end{array}\right] \\
Q_{f}=\left[\begin{array}{cc}
Q_{f}, & 0 \\
0 & Q_{f u}
\end{array}\right] \\
B=\left[\begin{array}{c}
B_{u} \\
B_{u}
\end{array}\right] \\
C=\left[\begin{array}{ll}
C_{v} & C_{u}
\end{array}\right]
\end{gathered}
$$

and for any $h \in \mathcal{H}$ one can write $h=h, \oplus h_{u}$ where $h, \in \mathcal{H}_{\text {, and }} h_{u} \in \mathcal{H}_{u}$. Now, by this construction one can also decompose $Y_{\beta}$ as

$$
Y_{\beta}=\left[\begin{array}{cc}
Y_{\beta,} & 0 \\
0 & Y_{\beta u}
\end{array}\right]
$$

In this way one can write the algebraic Riccati equation (A.R.E.) in two parts, corresponding to the stable and unstable subspace restrictions. Note that since $\mathcal{H}_{\mathrm{u}}$ is finite dimensional, the corresponding A.R.E. is a finite dimensional equation and thus, by Lemma 4.11 in Matson [2],

$$
\beta^{2} Y_{\beta u} C_{u}^{*} R_{f u}^{-1} C_{u} Y_{\beta u} \stackrel{S}{\rightarrow} B_{u} V B_{u}^{*}
$$

Thus, one needs only to show that this convergence holds for the A.R.E. corresponding to the stable subspace restriction. In other words, one needs to show that

$$
\beta^{2} Y_{\beta}, C_{s}^{*} R_{f}^{-1} C_{,} Y_{\beta,} \stackrel{s}{\rightarrow} B, V B_{i}^{*}
$$

Now, as shown in Curtain and Gibson $[10,3]$, one can write $Y_{\beta} h$ as

$$
Y_{\beta} h=\int_{0}^{\infty} T^{*}(s)\left(B V B^{*}+\beta^{-2} Q_{f}-\beta^{2} Y_{\beta} C^{*} R_{f}^{-1} C Y_{\beta}\right) T(s) h d s \quad \forall h \in \mathcal{H}
$$

where $T(t)$ is the stable semigroup generated by $A_{1}$. This is a different formulation from the one used by Matson [2] who used the identity

$\left\langle x_{0}, Y_{\mathcal{P}} x_{0}\right\rangle=\min _{u}\left\{\int_{0}^{\infty}\left\{\left(x(s),\left(B V B^{*}+\beta^{-2} Q_{J}\right) x(s)\right\rangle+\beta^{-2}\left\langle u(s), R_{f} u(s)\right\rangle\right] d s\right\}$

Also, Matson used the semigroup generated by the operator $A$ instead of the semigroup generated by the operator $A_{j}$. The expression for $Y_{p}$ used in this theorem yields the following inner product equation

$$
\left\langle Y_{\beta} h, k\right\rangle=\left\langle\int_{0}^{\infty} T^{*}(s) K_{\beta} T(s) h d s, k\right\rangle \quad \forall h, k \in \mathcal{H}
$$

where $K_{\beta}=\left(B V B^{*}+\beta^{-2} Q_{\rho}-\beta^{2} Y_{\beta} C^{*} R_{\rho}^{-1} C Y_{\beta}\right)$.

By continuity of the inner product, Curtain (see [3] page 93 ) shows that one can write

$$
\begin{gathered}
\left\langle Y_{\beta} h, k\right\rangle=\left\langle\int_{0}^{\infty} T^{*}(s) K_{\beta} T(s) h d s, k\right) \\
=\int_{0}^{\infty}\left\langle K_{\beta} T(s) h, T(s) k\right\rangle d s
\end{gathered}
$$

Now Matson [2] has shown that, for the case where the number of inputs equals the number of outputs, and under the assumptions of this development,

$$
\lim _{\beta \rightarrow \infty}\left\langle Y_{\beta} h, k\right\rangle=0
$$

so that, from the previous equality,

$$
\lim _{\beta \rightarrow \infty}\left\langle\int_{0}^{\infty} T^{*}(s) K_{p} T(s) h, k\right) d s=0 \quad \forall h, k \in \mathcal{H}
$$

Next, it will be shown that for $K_{\beta}$ positive semi-definite,

$$
\begin{aligned}
\int_{0}^{\infty} & \left\|K_{\beta} T(s) h\right\|^{2} d s \leq \\
& \left\{\int_{0}^{\infty}\left\langle K_{\beta} T(s) h, T(s) h\right) d s\right\}\left\{\int_{0}^{\infty}\left\langle K_{\beta}^{2} T(s) h, K_{\beta} T(s) h\right\rangle\right\}
\end{aligned}
$$

This will be needed in order to show that $\lim _{\beta \rightarrow \infty} \int_{0}^{\infty}\left\|K_{\beta} T(s) h\right\|^{2} d s=0$. Let $f(s)=T(s) h$, and define a pseudonorm on $f(s)$ as:

$$
\|f\|=[f, f]^{1 / 2}
$$

where $[f, y] \equiv \int_{0}^{\infty}\left\langle K_{\beta} f(s), y(s)\right\rangle d s$. A pseudonorm differs from a norm only in that $\|f\|$ can be zero for $f \neq 0$. Since $K_{\rho}$ is positive semi-definite, and not strictly positive, this can occur. Note that since $K_{\beta} \geq 0$, then $[f, f] \geq 0 \quad \forall f$. Thus, for any $\delta, \epsilon \in \Re$, and $f$ and $z$ in $\mathcal{H}$,

$$
0 \leq\|\delta f+\epsilon z\|^{2}
$$

which implies

$$
0 \leq \delta^{2}\|f\|+2 \delta \epsilon[f, z]+\epsilon^{2}\|z\|^{2}
$$

For $[f, z] \neq 0$, let $c=\frac{|f, z|}{\mid t, z] \mid}$ to yieid

$$
0 \leq \delta^{2}\|f\|^{2}+2 \delta|[f, z]|+\|z\|^{2}
$$

Now let $\delta=\frac{-\left\|e^{2}\right\|}{\|f\|^{2}}$. This then implies that:

$$
0 \leq \frac{[f, z]^{2}}{\|f\|^{2}}-\frac{2[f, z]^{2}}{\|f\|^{2}}+\|z\|^{2}
$$

This implies that

$$
|[f, z]| \leq\|f\|\|z\|
$$

Letting $f(s)=T(s) h$ and $z(s)=K_{\mathcal{A}} T(s) h$, from the last inequality one gets that

$$
\begin{aligned}
\int_{0}^{\infty} & \left\|K_{\beta} T(s) h\right\|^{2} d s \leq \\
& \left\{\int_{0}^{\infty}\left\langle K_{\beta} T(s) h, T(s) h\right\rangle d s\right\}\left\{\int_{0}^{\infty}\left\langle K_{p}^{2} T(s) h, K_{\beta} T(s) h\right\rangle\right\}
\end{aligned}
$$

Now, it can be shown that if $K_{\beta} \geq 0$ and if

$$
\lim _{\beta \rightarrow \infty}\left\langle\int_{0}^{\infty} T^{-*}(s) K_{\beta} T(s) h, k\right\rangle d s=0
$$

then, when $K_{\beta}$ is uniformly bounded independent of $\beta$, one gets that:

$$
\lim _{\beta \rightarrow \infty} \int_{0}^{\infty}\left\|K_{\beta} T(s) h\right\|^{2} d s=0
$$

It was shown that

$$
\begin{aligned}
\int_{0}^{\infty} & \left\|K_{\beta} T(s) h\right\|^{2} d s \leq \\
& \left\{\int_{0}^{\infty}\left(K_{\beta} T(s) h, T(s) h\right) d s\right\}\left\{\int_{0}^{\infty}\left\langle K_{\beta}^{2} T(s) h, K_{\beta} T(s) h\right\rangle\right\}
\end{aligned}
$$

However,

$$
\int_{0}^{\infty}\left\langle K_{\beta}^{2} T(s) h, K_{\beta} T(s) h\right\rangle d s \leq \int_{0}^{\infty}\left\|K_{\beta}\right\|^{3}\|T(s)\|^{2}\|h\|^{2} d s
$$


which yields

$$
\int_{0}^{\infty}\left\langle K_{\rho}^{2} T(s) h, K_{\rho} T(s) h\right\rangle d s \leq k^{3}\|h\|^{2} \int_{0}^{\infty}\|T(s)\|^{2} d s \equiv D<\infty
$$

where $\left\|K_{\beta}\right\| \leq k$. Thus, the integral is bounded independent of $\beta$. Since

$$
\lim _{\rho \rightarrow \infty}\left(\int_{0}^{\infty} T^{*}(s) K_{\rho} T(s) h, k\right) d s=0
$$

Then

$$
\begin{aligned}
\int_{0}^{\infty} & \left\|K_{\beta} T(s) h\right\|^{2} d s \leq \\
& \left\{\int_{0}^{\infty}\left\langle K_{\beta} T(s) h, T(s) h\right\rangle d s\right\}\left\{\int_{0}^{\infty}\left\langle K_{\beta}^{2} T(s) h, K_{\beta} T(s) h\right\rangle\right\}
\end{aligned}
$$

yields the result that

$$
\lim _{s \rightarrow \infty} \int_{0}^{\infty}\left\|K_{\beta} T(s) h\right\|^{2} d s=0
$$

With this last result, and using the fact that $K_{\rho}$ is uniformly bounded independent of $\beta$ (which is not integer valued) for $\beta$ sufficiently large, it can now be shown that:

$$
\lim _{\beta \rightarrow \infty}\left\|K_{\beta} h\right\|=0
$$

for all $h \in \mathcal{H}$, which is the desired convergence result. For any $t>0$, one gets that

$$
\begin{gathered}
\left|\left\|K_{\beta} T(t) h\right\|^{2}-\left\|K_{\beta} h\right\|^{2}\right|=\left|\int_{0}^{t} \frac{d}{d s}\left\|K_{\beta} T(s) h\right\|^{2} d s\right| \\
=2\left|\int_{0}^{t}\left\langle K_{\beta} A h, K_{\beta} T(s) h\right) d s\right| \\
\leq 2\left[\int_{0}^{t}\left\|K_{\beta} A h\right\|^{2} d s\right]^{1 / 2}\left[\int_{0}^{t}\left\|K_{\beta} T(s) h\right\|^{2} d s\right]^{1 / 2} \\
=2 t^{1 / 2}\left\|K_{\beta} A h\right\|\left[\int_{0}^{t}\left\|K_{\beta} T(s) h\right\|^{2} d s\right]^{1 / 2} \\
\leq 2 t^{1 / 2} M_{h}\left[\int_{0}^{t}\left\|K_{\beta} T(s) h\right\|^{2} d s\right]^{1 / 2} \\
\leq 2 t^{1 / 2} M_{h}\left[\int_{0}^{\infty}\left\|K_{\beta} T(s) h\right\|^{2} d s\right]^{1 / 2}
\end{gathered}
$$

Since $K_{\beta}$ is uniformly bounded, then $\left\|K_{\beta} A h\right\| \leq M_{h}$. Now, choose $t=t_{\beta}$ where

$$
t_{\beta}=\left\{\int_{0}^{\infty}\left\|K_{\beta} T(s) h\right\|^{2} d s\right\}^{-1 / 2}
$$

Notice that $t_{\beta} \rightarrow \infty$ as $\beta \rightarrow \infty$. Also, substituting this choice of $t=t_{\beta}$ into the inequality

$$
\|\| K_{\beta} T(t) h\left\|^{2}-\right\| K_{\beta} h \|^{2} \mid \leq 2 t^{1 / 2} M_{h}\left[\int_{0}^{\infty}\left\|K_{\beta} T(s) h\right\|^{2} d s\right]^{1 / 2}
$$
yields

$$
\left.\left|\left\|K_{\beta} T\left(t_{\beta}\right) h\right\|^{2}-\left\|K_{\beta} h\right\|^{2}\right| \leq 2 M_{h} \mid \int_{0}^{\infty}\left\|K_{\beta} T(s) h\right\|^{2} d s\right]^{1 / 4}
$$

This last inequality implies that

$$
\left\|K_{\beta} h_{1}^{\prime 2} \leq\right\| K_{\beta} T\left(t_{\beta}\right) h \|^{2}+2 M_{h}\left[\int_{0}^{\infty}\left\|K_{\beta} T(s) h\right\|^{2} d s\right]^{1 / 4}
$$

But since

$$
\begin{aligned}
\left\|K_{\beta} T\left(t_{\beta}\right) h\right\| & \leq\left\|K_{\beta}\right\|\left\|T\left(t_{\beta}\right)\right\|\|h\| \\
& \leq\left\|K_{\beta}\right\| M e^{\omega t_{\beta}}\|h\| \\
& \leq D\|h\| e^{\omega t_{\beta}}
\end{aligned}
$$

where $D$ is a constant depending on the bound of $K_{\beta}$ and the semigroup constant $M$. Since $\omega<0\left(T(t)\right.$ is a stable semigroup generated by $\left.A_{g}\right)$ and $t_{\beta} \rightarrow \infty$ as $\beta \rightarrow \infty$, then

$$
D\|h\| e^{w 1,} \rightarrow 0 \quad \text { as } \beta \rightarrow \infty
$$

This then yields from the inequality for $\left\|K_{\beta} h\right\|^{2}$ that

$$
\left\|K_{\beta} h\right\| \rightarrow 0 \text { as } \beta \rightarrow \infty \quad \forall h \in \mathcal{H}
$$

since it was established that $\int_{0}^{\infty}\left\|K_{\beta} T(s) h\right\|^{2} d s$ as $\beta \rightarrow \infty$. Thus, the desired convergence has been proven for $K_{\rho}$ being uniformly bounded independent of $\beta$, and for $K_{\beta}$ positive semi-definite. Q.E.D.

Since strong convergence is a sufficient condition for the LQG/LTR technique to be valid, then Theorem 2.1 gives a sufficient condition for the technique in terms of the operator $K_{\beta}$. Since $Y_{\beta}$ is bounded independent of $\beta$, it is reasonable to assume that $K_{\beta}$ is also. Therefore, the only restrictive assumption wouid appear to be the assumption that $K_{\beta}$ is positive semidefinite. Theorem 2.1 took a different approach than that found in Matson [2], and therefore may yield insights not before available that may allow the LQG/LTR to be extended to the entire class of problems considered in this research. The next section discusses why many authors have not been concerned with strong convergence (and thus have ignored the issue), and also provides another sufficient condition for strong convergence.

\section{Conditions for Strong Convergence}

This section will consider why it is difficult to prove strong convergence exists, and why many authors have avoided the issue when dealing with algebraic Riccati equations on infinite-dimensional spaces. The results of Lukes and Russell [11] will be applied to demonstrate that the A.R.E. of Theorem 2.1 has a bounded extension to the entire Hilbert space $\mathcal{H}$, which is why most authors have considered the A.R.E. to be defined on the entire Hilbert space. A bounded extension $\mathcal{B}_{\boldsymbol{\varepsilon}}$ is a bounded linear operator that equals an operator $\Xi$, the operator to be extended, on $D(\Xi)$, and which is defined on the entire Hilbert space $\mathcal{H}$ containing $D(\Xi)$.

The difficulty in proving that strong convergence exists, is the fact that the algebraic Riccati equation (A.R.E.) is only defined on $\mathrm{D}\left(A^{*}\right)$. The equation has been considered by many to be defined on the entire Hilbert space $\mathcal{H}$ because of the following lemma from Lukes and Russell [11].

LEMMA 3.1: The operator $\left(Y_{\beta} A^{*}+A Y_{\beta}\right)$ associated with the A.R.E. given by

$$
\begin{array}{r}
<\left(Y_{\beta} A^{*}+A Y_{\beta}\right) h, k>+<B V B^{*} h, k>+<\beta^{-2} Q_{l} h, k> \\
=\beta^{2}<Y_{\beta} C^{*} R_{\jmath}^{-1} C Y_{\beta} h, k>\quad \forall h, k \in D\left(A^{*}\right)
\end{array}
$$

has a bounded linear extension to all of $\mathcal{H}$ for each $\beta$.

Proof: (see Lukes and Russell [11]). From Lemma 3.1, for $h, k \in D\left(A^{*}\right)$, one has that

$$
\left\langle A^{*} h, Y_{\beta} k\right\rangle+\left\langle Y_{\beta} h, A^{*} k\right\rangle=\left\langle B_{\varepsilon} h . k\right\rangle
$$

where $B_{\varepsilon}$ is a bounded linear operator defined on all of $\mathcal{H}$, and $B_{\varepsilon}=\beta^{2} Y_{\beta} C^{*} R_{j}^{-1} C Y_{\beta}-\left(B V B^{*}+Q_{J} / \beta^{2}\right)$. Since $B_{\varepsilon}$ is defined on all of $\mathcal{K}$, then it is true that $\left(Y_{\beta} A^{*}+A Y_{\beta}\right)$ has a bounded extension equal to $B_{\mathcal{E}}$ on all of $\mathcal{H}$ for each $\beta$. One should note that $B_{\varepsilon}$ is dependent on $\beta$, so that there is not necessarily a $\mathcal{B}_{\mathcal{E}}$ that satisfies the A.R.E. of Lemma 3.1 for all B. Q.E.D.

It is not known whether or not one can show that $\mathcal{B}_{\mathcal{E}}$ of Lemma 3.1 is uniformly bounded for all $\beta$. If one could show that to be true, then $K_{\beta}$ of Theorem 2.1 would be uniformly bounded. Another useful insight comes from Gibson [10], who demonstrates that $Y_{\beta}$ maps $D\left(A^{*}\right)$ onto $D(A)$. This fact allows one to write $\left\langle Y_{\beta} h, A^{*} k\right\rangle$ as $\left\langle A Y_{\beta} h, k\right\rangle$ so that the A.R.E. can be written as

$$
\begin{array}{r}
<Y_{\beta} A^{*} h, k>+<A Y_{\beta} h, k>+<B V B^{*} h, k>+\left\langle Q_{f} / \beta^{2} h, k>\right. \\
=\beta^{2}<Y_{\beta} C^{*} R_{f}^{-1} C Y_{\beta} h, k>\quad \forall h, k \in D\left(A^{*}\right)
\end{array}
$$

and the strong convergence of $Y_{\beta}$ to 0 [2], for the class of systems in Theorem 2.1, yields

$$
\lim _{\beta \rightarrow \infty} \beta^{2}\left\langle Y_{\beta} C^{*} R_{j}^{-i} C Y_{\beta}^{\prime} h, k\right\rangle=\left\langle B V B^{*} h, k\right\rangle \quad \forall h, k \in D\left(A^{*}\right)
$$

Therefore, the question one needs to ask is, when does $\beta^{2} Y_{\theta} C^{*} R_{f}^{-1} C Y_{\beta} \rightarrow$ $B V B^{*}$ strongly as $\beta \rightarrow \infty$ ? The following lemma gives a sufficient condition for this to happen.

LEMMA 3.2: For the assumptions of Theorem 2.1, if $\left\langle\beta^{2} Y_{\beta} C^{*} R_{f}^{-1} C Y_{\rho} h, h\right\rangle$ is a monotone (either increasing or decreasing) sequence for increasing $\beta$, then

$$
\beta^{2} Y_{\beta} C^{*} R_{j}^{-1} C Y_{\beta} \stackrel{s}{\rightarrow} B V B^{*}
$$

where convergence is strong.

Proof: Only the case of $\beta^{2} Y_{\beta} C^{*} R_{\rho}^{-1} C Y_{\beta}$ being monotonically increasing will be considered. If $\beta^{2} Y_{\beta} C^{*} R_{\rho}^{-1} C Y_{\beta}$ is monotonically increasing, then, since the limit of Equation (11) exists, it must be true that $\left[B V B^{*}-\right.$ $\left.\beta^{2} Y_{\beta} C^{*} R_{f}^{-1} C Y_{\beta}\right]$ is positive $\forall \beta$ and $\forall h \in D\left(A^{*}\right)$. Then Theorem 3.6 in 
[12], page 453, yields that $\beta^{2} Y_{\beta}^{\prime} C^{*} R_{f}^{-1} C Y_{\beta} \rightarrow B V B^{*}$, with convergence being strong. A similar result applies if $\beta^{2} Y_{\beta} C^{*} R_{f}^{-1} C Y_{\beta}$ is monotonically decreasing. In that case the operator $\left[\beta^{2} Y_{\beta} C^{*} R_{\delta}^{-1} C Y_{\beta}-B V B^{*}\right]$ is positive, and again [12] yields that strong convergence exists. Q.E.D.

It is not known whether or not this sufficient condition can be demonstrated for the entire class of systems described in Section 1. However, it is believed that it will occur in many problems. As a designer, one must evaluate $\beta^{2} Y_{\beta} C^{*} R_{f}^{-1} C Y_{\beta}$ on a problem by problem basis to determine if it is monotone, to see if the LQG/LTR technique can be used confidently. This may not be easy to do. Even if the operator is not monotone, the LQG/LTR technique may still work since this is only a sufficient condition. The next lemma gives one sufficient design condition for $\beta^{2} Y_{\beta} C^{*} R_{f}^{-1} C Y_{\beta}$ to be monotone. In the lemma, $V$ and $R_{f}$ will be chosen to be identity operators. These choices are made to simplify the details of the proof, which only requires that $V$ and $R_{f}$ be as described in Section 1 . Other assumptions needed in the next lemma are that the inputs $u$ be elements of $L_{2}$ space, and that the state variable $x$ be bounded away from zero. The assumption on $u$ is not very restrictive for real problems. The assumption on $x$ implies that the system has stochastic controllability so that $x$ can be bounded away from zero by adding white noise. Two additional assumptions involve the operators $B^{*}$ and $C$. These assumptions do not have physical significance, but rather are chosen in order to make the proof work.

LEMMA 3.3: Make the assumptions made in Theorem 2.1 (except for the ones for $K_{\theta}$ ). Let $Q_{f}$ be a bounded positive linear operator with a bounded square root $Q_{f}^{1 / 2}$, and let the positive definite matrix $\mathrm{V}=1$, let $R_{f}=1$, and let

$$
\begin{gathered}
\int_{0}^{\infty}\|u(s)\|^{2} d s \leq M \quad \forall u \in U \\
\int_{0}^{\infty}\|x(s)\|^{2} d s \geq \varepsilon>0 \quad \forall x \in \mathcal{H}
\end{gathered}
$$

and let $B^{*}$ be such that its inverse exists (not necessarily typical of physical problems) so that

$$
\int_{0}^{\infty}\left\|B^{*} x(s)\right\|^{2} d s \geq k \int_{0}^{\infty}\|x(s)\|^{2} d s \quad, k>0
$$

Finally, let $C$ be a unitary operator. Then under these conditions,

$$
\beta^{2} Y_{\beta} C^{*} R_{f}^{-1} C Y_{\beta}
$$

is monotone increasing.

Proof: Since $Y_{\beta}$ satisfies the A.R.E., then it can be expressed [3] as

$$
\left.\left\langle x_{0}, Y_{\beta} x_{0}\right\rangle=\min _{u} \int_{0}^{\infty}[<x(s), \theta x(s)\rangle+\beta^{-2}<u(s), R_{f} u(s)>\right] d s
$$

where $x_{0} \in \mathcal{H}$ and $\theta=B B^{*}+Q_{\zeta} / \beta^{2}$. This then yields

$\left\langle x_{0}, \beta Y_{\beta} x_{0}\right\rangle=\min _{u} \int_{0}^{\infty}\left[\langle x(s), \beta \theta x(s)\rangle+\beta^{-1}\left\langle u(s), R_{f} u(s)\right\rangle\right] d s$

As demonstrated by [3], the minimum is attained with $u=-K, x$. Let $u^{*}$ and $x^{*}$ be the solutions obtained by performing the minimization. Then one gets

$$
\left.\left\langle x_{0}, \beta Y_{\beta} x_{0}\right\rangle=\int_{0}^{\infty}\left[\left\langle x^{*}, \beta \theta x^{*}\right\rangle+\beta^{-1}<u^{*}, R_{f} u^{*}\right\rangle\right] d s
$$

Substituting for $\theta$ then gives

$$
\begin{aligned}
& \left\langle x_{0}, \beta Y_{\beta} x_{\circ}\right\rangle=\int_{0}^{\infty}\left[\left\langle x^{*}, \beta B B^{*} x^{*}\right\rangle+1 / \beta<x^{*}, Q_{f} x^{*}\right\rangle+ \\
& \left.1 / \beta<u^{*}, R_{f} u^{*}>\right] d s
\end{aligned}
$$

which is equivalent to

$$
\begin{array}{r}
\left\langle x_{0}, \beta Y_{\beta} x_{0}\right\rangle=\int_{0}^{\infty} \beta\left\|B^{*} x^{*}\right\|^{2} d s+1 / \beta \int_{0}^{\infty}\left\|Q_{f}^{1 / 2} x^{*}\right\|^{2} d s+ \\
1 / \beta \int_{0}^{\infty}\left\|R_{j}^{1 / 2} x^{*}\right\|^{2} d s
\end{array}
$$

Define this inner product as $H(\beta)=\left\langle x_{0}, \beta Y_{\beta} x_{0}\right\rangle$. This inner product will be an increasing function of $\beta$ if its derivative $H^{\prime}(\beta)$ is positive, which occurs if

$$
\begin{array}{r}
H^{\prime}(\beta)=\int_{0}^{\infty}\left\|B^{*} x^{*}\right\|^{2} d s-1 / \beta^{2} \int_{0}^{\infty}\left\|Q_{f}^{1 / 2} x^{*}\right\|^{2} d s- \\
1 / \beta^{2} \int_{0}^{\infty}\left\|R_{f}^{1 / 2} x^{*}\right\|^{2} d s
\end{array}
$$

is positive. The fact that $Q_{f}^{1 / 2}$ is a bounded operator and the assumptions of the lemma yield

$$
\begin{gathered}
H^{\prime}(\beta) \geq K \int_{0}^{\infty}\left\|x^{*}\right\|^{2} d s-\frac{\tilde{K}}{\beta^{2}} \int_{0}^{\infty}\left\|x^{*}\right\|^{2} d s-\frac{M}{\beta^{2}} \int_{0}^{\infty}\left\|x^{*}\right\|^{2} d s \\
H^{\prime}(\beta)>K \varepsilon-\frac{\tilde{K} \varepsilon}{\beta^{2}}-\frac{M}{\beta^{2}} \\
H^{\prime}(\beta)>K \varepsilon-\frac{\tilde{K} \varepsilon+M}{\beta^{2}}
\end{gathered}
$$

which, for $\beta$ sufficiently large, is positive $\forall x_{0} \in \mathcal{H}$. Now, since $\beta Y_{\beta}$ is selfadjoint, then it is also normal (i.e. $\beta Y_{\beta} \beta Y_{\beta}^{*}=\beta Y_{\beta}^{*} \beta Y_{\beta}$ ). This yields that the sqaure root is normal [13]. Then by Theorem 5.23 .15 in [14],

$$
\left\|\beta^{1 / 2} Y_{\beta}^{1 / 2} x_{o}\right\|^{2}=\left\|\beta Y_{\beta} x_{0}\right\|=\left\|\left(\beta^{1 / 2} Y_{\beta}^{1 / 2}\right)^{2} x_{0}\right\|
$$

Thus, if $\left\langle x_{0}, \beta Y_{\beta} x_{0}\right\rangle$ increases as $\beta$ increases (which is proven in [2]), then

$\left\|\beta^{1 / 2} Y_{\beta}^{1 / 2} x_{0}\right\|^{2}$ increases, as does $\left\|\beta Y_{\beta} x_{0}\right\|^{2}$. This gives that

$<\beta Y_{\beta} x_{o}, \beta Y_{\beta} x_{o}>$ is a monotonically increasing function for $\beta$ sufficiently large.

Now, let $C$ be a unitary operator. Then for $R_{f}=I$,

$$
\begin{aligned}
\left\langle\beta^{2} Y_{\beta} C^{*} R_{f}^{-1} C Y_{\beta} x_{0}, x_{0}\right\rangle= & \left\langle R_{f}^{-1 / 2} C \beta Y_{\beta} x_{0}, R_{f}^{-1 / 2} C \beta Y_{\beta} x_{0}\right\rangle \\
= & \left\langle C \beta Y_{\beta} x_{0}, C \beta Y_{\beta} x_{0}\right\rangle \\
= & \left\langle C^{*} C \beta Y_{\beta} x_{0}, \beta Y_{\beta} x_{0}\right\rangle \\
= & \left\langle\beta Y_{\beta} x_{0}, \beta Y_{\beta} x_{0}\right\rangle
\end{aligned}
$$

so that for $\beta$ sufficiently large, $\beta^{2} Y_{\beta} C^{\cdot} R_{f}^{-1} C Y_{\beta}$ is a monotonically increasing operator. Q.E.D.

The problem with Lemma 3.3 is that the conditions are not physically motivated. In the case where the input space is finite-dimensional, $\left\|R_{f}^{-1 / 2} u(s)\right\|$ will be bounded and stochastic controllability would imply that the states can be bounded away from zero by adding white noise. However, one cannot usually expect to satisfy the condition

$$
\int_{0}^{\infty}\left\|B^{*} x(s)\right\|^{2} d s \geq k \int_{0}^{\infty}\|x(s)\|^{2} d s
$$

or to have the operator $C$ be a unitary operator. The lemma does give some added insight into how to approach showing that $\beta^{2} Y_{\beta} C^{*} R_{f}^{-1} C Y_{\beta}$ is a monotonically increasing operator for other classes of problems. For example, it may be possible to show that, for certain problems,

$$
\frac{\int_{0}^{\infty}\left\|Q_{f}^{1 / 2} x^{*}\right\|^{2} d s+\int_{0}^{\infty}\left\|R_{f}^{1 / 2} x^{*}\right\|^{2} d s}{\int_{0}^{\infty}\left\|B^{*} x(s)\right\|^{2} d s} \leq K
$$

i.e., it is uniformly bounded $\forall x$ and $u$. This would result in $H^{\prime}(\beta)$ being positive, so that (using a proof similar to that of Lemma 3.3) one can show there exists a $\beta$ such that $\left\langle\beta Y_{\beta} x_{0}, \beta Y_{\beta} x_{0}\right\rangle$ is monotone. Also, it may be possible to prove that $\left\langle C \beta Y_{\beta} x_{0}, C \beta Y_{\beta} x_{0}\right\rangle$ is monotone for arbitrary $C \in \mathcal{L}\left(\mathcal{H} ; \Re^{N}\right)$, given that $\left\langle\beta Y_{\beta} x_{0}, \beta Y_{\beta} x_{0}\right\rangle$ is monotone.

However, if $\beta^{2} Y_{\beta} C^{*} R^{-1} C Y_{\beta}$ is not monotone, then nothing can be said about whether or not the LQG/LTR technique is valid, because Lemma 3.2 only provides a sufficient condition, as opposed to necessary and sufficient. Since a set of physically meaningful conditions was not obtained to ensure the desired strong convergence, one may try to apply the procedure in an ad hoc fashion, or one may try to use another approach to achieve robustness. One alternative is to try to approximate the LQG/LTR procedure. The next section will look at using the approximation approach of Banks [7] as a way to approximate the LQG/LTR procedure.

\section{LQG/LTR Approximation}

The LQG/LTR approach to robust LQG controller design makes the robustness recovery a part of the A.R.E. In this way one "tunes" the filter in order to regain the guaranteed robustness of the LQ regulator [1], or one can use Kwakernaak and Sivan's [5] dual procedure, depending on on the point in the loop where one chooses to enhance robustness. If one chooses to enhance robustness at the input to the plant, then one can use Doyle and 
Stein's approach [15] to recover the loop transfer function of the regulator at the plant input. If one enhances robustness at the plant output, one can use the dual procedure of Kwakernaak and Sivan [5] to recover the loop transfer function associated with the Kalman filter at the plant output. However, if one were able to apply the LQG/LTR technique validly for the clase of systems considered in this paper, one would still have to solve an infinite-dimensional A.R.E., and then use some technique to approximate the desired "robust" compensator. The problem is, that it is not known if the LQG/LTR technique can be validly applied to the entire class of distributed systems considered in this paper.

One way to approach designing a robust compensator might be to approximate the infinite dimensional A.R.E. with a sequence of finite dimensional A.R.E.s such that the sequence of solutions converges to the infinite dimensional A.R.E. solution, as done in [7]. In this way, one could approximate the desired solution, and perform robustness enhancement on the approximation. Thus, one could possibly approximate the LQG/LTR technique for the entire class of systems.

In order to make direct use of the results of [7], the dual robustness recovery procedure of Kwakernaak and Sivan will be considered. By duality the type of results obtained are applicable to the robustness recovery procedure of Doyle and Stein. For that approach, one merely has to consider the dual A.R.E. and the operators associated with it. The development that follows involves the A.R.E. associated with the $L Q$ regulator gain operator $K_{c}$. The operator II used by Banks is the operator that solves the regulator A.R.E.

Following the presentation by Banks [7], and using his notation, $\Pi \in$ $\mathcal{L}(\mathcal{H})$ is a solution of the A.R.E. given by

$$
A^{*} \Pi+\Pi A+Q_{c}-\Pi B R_{c}^{-1} B^{*} \Pi=0
$$

if $\Pi$ maps $D(A)$ into $D\left(A^{*}\right)$ and if $\Pi$ satisfies the A.R.E. on $D(A)$.

One thing to note is that, this A.R.E. is actually an inner product equation. The inner product notation has been dropped in order to simplify the writing of the equations. Kwakernaak and Sivan's approach to robustness recovery involves modifying the state cost weighting operator $Q_{e}$, by adding the additional cost term $q^{2} C^{*} V C$ to $Q_{c}$. Then, as $q \rightarrow \infty$, one is able to asymptotically recover the loop transfer function associated with the Kalman filter, at the plant output. The problem with this LQG/LTR technique is that, in order to converge to the guaranteed stability robustness of the filter, one must show that

$$
\lim _{i \rightarrow \infty} q^{2}\left\langle\Pi B R_{e}^{-1} B^{*} \Pi h, k\right\rangle=\left\langle C^{*} V C h, k\right\rangle \quad \forall h, k \in D(A)
$$

implies strong convergence [2]. Matson [2] has proven the dual case (and thus this case by duality) when $A^{*}$ is a finite-dimensional (i.e. bounded) operator, and also when $R(B) \cup R\left(Q_{f}\right)$ is contained in a finite-dimensional space spanned by a finite number of eigenfunctions of $A^{*}$.

Following the development of Banks [7], the infinite-dimensional state space problem given by

$$
\begin{gathered}
\dot{x}=A x+B u \quad x_{\circ} \in D(A) \\
y=C x
\end{gathered}
$$

where $x \in \mathcal{H}$, can be redefined by projecting it onto a finite-dimensional subspace of $\mathcal{H}$ (denoted $\mathcal{H}_{k}$ ) by means of an orthogonal projection $P^{k}$ such that $P^{k}: \mathcal{K} \rightarrow \mathcal{K}_{k}$. A projection $P^{k}$ is said to be orthogonal if its range and null space are orthogonal, if $P^{k} P^{k}=P^{k}$, and if each $h \in \mathcal{H}$ can be written uniquely as $h=r+n$ where $r$ is in the range of $P^{k}$, and $n$ is in the null space of $P^{k}$. Also, an orthogonal projection is continuous.

This projection is used to define the operators $A^{k}, B^{k}, C^{k}$ and $Q_{c}^{k}$ as follows:

$$
\begin{gathered}
P^{k} A=A^{k} \in \mathcal{L}\left(\mathcal{H}_{k}\right) \\
P^{k} B=B^{k} \in \mathcal{L}\left(\Re^{N} ; \mathcal{H}_{k}\right) \\
P^{k} C=C^{k} \in \mathcal{L}\left(\mathcal{H}_{k} ; \Re^{N}\right) \\
P^{k} Q_{\varepsilon}=Q_{c}^{k} \in \mathcal{L}\left(\mathcal{H}_{k}\right)
\end{gathered}
$$

Using these operators, the family of finite-dimensional regulator problems described by

$$
\begin{gathered}
\dot{x}=A^{k} x+B^{k} u ; \quad x(0)=P^{k} x_{0} \\
y=C^{k} x
\end{gathered}
$$

with associated cost functional given by

$$
J^{k}=\int_{0}^{\infty}\left[\left\langle Q_{c}^{k} x, x\right\rangle+\left\langle R_{c} u, u\right\rangle\right] d t
$$

can be considered. It will also be necessary to make the following assumptions on each finite-dimensional problem:
1. For each $x_{0} \in \mathcal{H}_{k}$, there exists an admissible control $u \in L^{2}(0, \infty ; U)$ such that the cost functional $J^{k}$ is finite

2. (a) $Q_{c}$ is positive semi-definite on $\mathcal{H}$, as is $P^{k} Q_{c}$ on $\mathcal{H}_{k}$ for all $\mathrm{k}$ (i.e. $\left\langle Q_{c} x, x\right\rangle \geq 0 \quad \forall x \in \mathcal{H}$ ) and $\left\langle Q_{c}^{k} x, x\right\rangle \geq 0 \quad \forall x \in \mathcal{H}_{k}$

(b) $R_{c}$ is positive on the input space $\mathrm{U}$ (i.e. $\left\langle R_{c} u, u\right\rangle>0 \forall u \in$ $U$ )

3. (a) $P^{k} \rightarrow I$ as $k \rightarrow \infty$ in the sense that, for any $x \in \mathcal{H}, P^{k} x \rightarrow x$ as $k \rightarrow \infty$

(b) $T^{k}(t) P^{k} x \rightarrow T(t) x$ as $k \rightarrow \infty$, where $T(t)$ is the semigroup generated by the operator $A$

(c) $T^{* k}(t) P^{k} x \rightarrow T^{*}(t) x$ as $k \rightarrow \infty$, where $T^{*}(t)$ is the dual semigroup generated by $A^{*}$

(d) $B^{k} u \rightarrow B u$ and $B^{* k} x \rightarrow B^{*} x$ as $k \rightarrow \infty$

(e) $Q_{c}^{k} P^{k} \rightarrow Q_{c} x$ as $k \rightarrow \infty$

Assumption 1 is a standard LQG assumption and is satisfied by our assumptions of stabilizability and detectability. Specifically, it is assumed that $(A, B)$ and $(A, G)$ are stabilizable, and $(A, C)$ and $\left(A, Q_{c}^{1 / 2}\right)$ are detectable. Assumption 2 is made in order to use the results of [2] and it is not very restrictive in applications. If assumption 1 holds, then the optimal control for each finite-dimensional problem is given by

$$
u^{k}=-R_{c}^{-1} B^{* k} \Pi^{k} x
$$

where $\Pi^{k} \in \mathcal{L}\left(\mathcal{H}_{k}\right)$ is the unique nonnegative self-adjoint solution of the A.R.E. on $\mathcal{H}_{k}$ given by

$$
A^{* k} \Pi^{k}+\Pi^{k} A^{k}+Q_{c}^{k}-\Pi^{k} B^{k} R_{c}^{-1} B^{* k} \Pi^{k}=0
$$

Assumptions 3 a-f can be satisfied by many approximation schemes, for example modal approximation [7], so that these assumptions are not overly restrictive. In fact, $3(\mathrm{a})$ implies $3(\mathrm{~d})$ since $B$ is a bounded linear operator. The assumption that $(A, B)$ is exponentially stabilizable along with the assumption that $\left(A, Q_{c}^{1 / 2}\right)$ is exponentially detectable (as is done in this research) allows the hypothesis of Theorem 2.2 of [7] to be satisfied for a large class of distributed parameter systems using several appproximation schemes (i.e. modal, spline, Ritz, etc. [16]). The following theorem is taken from [7].

THEOREM 4.1: Suppose that the previous Assumptions 1 thru 3(e) hold. Let $Q_{c} \geq 0, R_{c}>0$, and let $\Pi^{k}$ denote the unique nonnegative self-adjoint Riccati solution on $\mathcal{H}_{k}$. Also assume that a unique nonnegative self-adjoint Riccati operator II exists for the problem defined on $\mathcal{H}$. Let $S^{k}(t)$ and $S(t)$ be the semigroups generated by $\left(A^{k}-B^{k} R_{c}^{-1} B^{* k} \Pi^{k}\right)$ and $\left(A-B R_{c}^{-1} B^{*} \Pi\right)$ on $\mathcal{H}_{k}$ (a finite-dimensional subspace of the Hilbert space $\mathcal{K}$ ) and $\mathcal{H}$ respectively, and let $\|S(t) x\| \rightarrow 0$ as $t \rightarrow \infty \forall x \in \mathcal{H}$. If there are two positive constants $\left(M_{1}, M_{2}\right)$ and $\omega<0$ that are all independent of both $\mathrm{k}$ and $\mathrm{t}$ such that

i) $\left\|S^{k}(t)\right\| \leq M_{1} e^{\omega t} \quad \forall t \geq 0$ and $k=1,2, \ldots$,

ii) $\left\|\Pi^{k}\right\| \leq M_{2}$

then, as $k \rightarrow \infty$,

a) $\Pi^{k} P^{k} x \rightarrow \Pi x \quad \forall x \in \mathcal{H}$

b) $S^{k}(t) P^{k} x \rightarrow S(t) x \quad \forall x \in \mathcal{H}$

where the convergence is uniform in $t$ on bounded subsets of $[0, \infty)$, and

$$
\|S(t)\| \leq M_{1} e^{\omega t} \quad \forall t \geq 0, \quad \omega<0
$$

Proof: See Banks [7] page 695.

Note that, since the $L Q$ regulator generates a stable semigroup [3], then $\|S(t) x\| \rightarrow 0$ as $t \rightarrow \infty$. Thus, using this approach, one can approximate the solution of the infinite-dimensional A.R.E. with a sequence of finite-dimensional A.R.E.'s. Banks [7] demonstrates that the hypothesis of Theorem 4.1 can be satisfied by many popular approximation schemes (such as modal, spline, etc). Once the solution is approximated closely enough, then one could apply the LQG/LTR technique using the finitedimensional A.R.E. The drawback to this approach is that guaranteed sta. 
bility margins to be approached asymptotically are only with respect to the finite-dimensional model, not the infinite-dimensional model. However, models are always an approximation of reality. The idea is to choose a model that matches the real world well enough so that the compensator's robustness can tolerate the model uncertainty. One also wants robustness to deal with model perturbations for the case when a system changes during operation. Even distributed parameter system models are approximations, but they are often used because they are better approximations for some systems than finite-dimensional models. Since Banks' approach involves approximating the distributed parameter system solution by approximating the associated infinite-dimensional A.R.E. with a sequence of finite-dimensional A.R.E's., the development of this section can be viewed as an approximation of the LQG/LTR technique.

If perturbations are modeled as finite-dimensional, then this approach can be used to get a desirable compensator. If not, then this approach provides added robustness with respect to a finite-dimensional model which can be made arbitrarily close to the system under consideration. In this way one can say he is approximating the LQG/LTR technique on the infinitedimensional system. However, for systems for which the LQG/LTR is not known to be valid, it is not clear what type of robustness is achieved in the limit as the order $k$ of the approximation goes to $\infty$. It is believed that the approximation technique will yield desirable results. However, the LQG/LTR technique is just one way to achieve robustness.

\section{Summary}

This paper presents sufficient conditions that allow the LQG/LTR technique to be extended to a class of problems of interest. In particular, Theorem 2.1 gives conditions based on the operator $K_{\beta}=\left(B V B^{*}+\beta^{-2} Q_{j}-\right.$ $\left.\beta^{-2} Y_{\beta} C^{*} R_{f}^{-1} C Y_{\beta}^{\prime}\right)$. It is shown that, if $K_{\beta}^{\prime}$ is uniformly bounded independent of $\beta$, and if $K_{\beta}$ is positive semi-definite, then the LQG/LTR technique is valid for the class of problems that satisfy the assumptions of Section 1. Theorem 2.1 is based on a different development than that found in Matson's work [2], and may provide insight as to how to extend the LQG/LTR technique to the entire class of problems without any conditions on $K_{\beta}$.

Section 3 discussed the reason many people have not considered strong convergence an issue. Since the A.R.E. has a bounded extension to the entire Hilbert space $\mathcal{H}$, some have simply assumed strong convergence. It is shown in Section 3, that weak convergence does exist for the class of problems considered in this research. Section 3 also has a second set of sufficient conditions that allow the LQG/LTR technique to be extended. The conditions are not physically meaningful, but Lemma 3.3 provides yet another approach to solving the problem of extending the LQG/LTR technique.

Since the technique has not been extended to the entire class of problems considered in this paper, an approximation of the technique is developed, based on the work of Banks [7]. Section 4 shows how to approximate the LQG/LTR technique by approximating the infinite-dimensional A.R.E. with a sequence of finite-dimensional A.R.Es. Since the LQG/LTR technique is valid for finite-dimensional systems, then one can use the finitedimensional A.R.E.'s. to approximate the technique.

\section{References}

[1] J. C. Doyle and G. Stein, "Multivariable Feedback Design: Concepts for a Classical/Modern Synthesis," IEEE Transactions on Automatic Control, vol. AC-26, no. 1, pp. 4-16, 1981

[2] C. L. Matson, The Linear Quadratic Gaussian / Loop Transfer Recovery Technique for a Class of Distributed Parameter Systems. PhD thesis, Air Force Institute of Technology, Wright-Patterson AFB, Ohio, December 1986

[3] R. F. Curtain and A. J. Pritchard, Infinite Dimensional Linear System Theory. Springer-Verlag, New York, 1978.

[4] J. M. Schumacher, Dynamic Feedback in Finite- and InfiniteDimensional Linear Systcms. PhD thesis, Mathematisch Centrum, M. C. Tracts No. 143, Amsterdam, 1982.

[5] H. Kwakernaak and R. Sivan, Linear Optimal Control Systems. Wiley Interscience, New York, 1972.

[6] A. E. Taylor and D. C. Lay, Introduction to Functional Analysis. Wiley and Sons, second ed., 1980.

[7] H. T. Banks and K. Kunisch, "The Linear Regulator Problem for Parabolic Systems," SIAM Journal of Control and Optimization vol. 22, pp. 684-698, September 1984
[8] C. A. Jacobson, "Some Aspects of the Structure and Stability of a Class of Linear Distributed Systems," Master's thesis, Rensselaer Polytechnic Institute, Troy, New York, May 1984.

[9] R. F. Curtain, "Equivalence of Input-Output Stability and Exponential Stability for Infinite Dimensional Systems, ${ }^{n}$ Tech. Rep. TW-276, Mathematics Institute Rijksuniveriteit Groningen, P.O. Box 800, 9700 AV Gronigen, The Netherlands, August 1987.

[10] J. S. Gibson, "The Riccati Integral Equations for Optimal Control Problems on Hilbert Space, SIAM Journal of Control and Optimization, vol. 17, pp. 537-565, January 1979.

[11] D. L. Lukes and D. L. Russell, "The Quadratic Criterion for Distributed Systems," SIAM Journal of Control and Optimization, vol. 7, pp. 101-121, 1969

[12] T. Kato, Periurbation Theory for Linear Operators. New York: Springer-Verlag, 1966.

[13] P. R. Halmos, A Hilbert Space Problem Book. Princeton: D. Van Nostrand Co., Inc., 1967.

[14] A. W. Naylor and G. R. Sell, Linear Operator Theory in Engineering and Science. New York: Springer-Verlag, 1982.

[15] G. Stein and J. C. Doyle, "Robustness with Observers," IEEE Transactions on Automatic Control, vol. AC-24, pp. 607-611, August 1979.

[16] 1. M. Gelfand and S. V. Fomin, Calculus of Variations. Englewood Cliffs, New Jersey: Prentice-Hall, Inc., 1963. 